\title{
Search for an enhanced emission of neutrinos from the Southern Sky with the ANTARES telescope
}

\author{
Luigi Antonio Fusco*i \\ Dipartimento di Fisica e Astronomia dell'Università di Bologna, Viale Berti-Pichat 6/2, 40127, \\ Bologna, Italy. \\ INFN - Sezione di Bologna, Viale Berti-Pichat 6/2, 40127, Bologna, Italy.
}

E-mail: Ifusco@bo.infn.it

\begin{abstract}
Compelling evidence of the existence of cosmic neutrinos has been reported by the IceCube collaboration. Some features of this signal could be explained by a Northern/Southern sky asymmetry of the flux. This possible asymmetry could be related to the presence of the bulk of our Galaxy in the Southern sky.

The ANTARES detector, located in the Mediterranean Sea, is currently the largest operating under-water neutrino telescope. Its effective area and good exposure to the Southern sky allows to constrain an enhanced muon neutrino emission from extended sources.

Two signal regions are defined: one around the largest accumulation of events from the IceCube High Energy Starting Events and one surrounding the Galactic Plane area; the background from atmospheric events is estimated looking at data from off-zones for which ANTARES has the same exposure as for the signal region. The ANTARES sensitivity to such a flux has been computed and the results of the analysis of data from 2007 to 2013 are presented.
\end{abstract}

The 34th International Cosmic Ray Conference,

30 July- 6 August, 2015

The Hague, The Netherlands

\footnotetext{
* Speaker.

†n behalf of the ANTARES Collaboration.
} 


\section{Introduction}

The scientific goal of the ANTARES neutrino telescope [1] is to detect high energy neutrinos of cosmic origin. The IceCube Collaboration has announced the observation of a cosmic neutrino signal in the High Energy Starting Events (HESE) analysis [2] of two years of data with the complete detector. The purely atmospheric expectation is rejected at a level of $5.7 \sigma$ with the inclusion of a third year of data [3].

The IceCube neutrino signal is reported to be compatible with a cosmic flux which is equally distributed in the three neutrino flavours [4] and isotropic in the neutrino arrival direction. However, some possible enhancement of the neutrino flux from the Southern with respect to the Northern hemisphere has been underlined for example in [5]. The low energy extension of the IceCube HESE analysis [6] shows a steepening of the energy spectrum of the cosmic signal and more events in the low energy part of the signal. Even if the IceCube detector is much larger in volume, the effective area of ANTARES in the region between 20 and $50 \mathrm{TeV}$ in the Southern Sky is similar to that of IceCube: some IceCube-like signal events could be observed by ANTARES, too.

Because of the nature of the IceCube analysis, based on vetoing techniques to detect downwardgoing events [7], the signal is mainly distributed in the shower channel: the directional resolution for these events is poor and the signal appears as an all sky flux. Since ANTARES is located in the Mediterranean Sea, the Southern sky is accessible to the detector in up-going tracks, for which an extremely good angular resolution can be achieved. For this reason a diffuse flux in the shower channel for IceCube might appear as an ensemble of individual point sources in ANTARES or a region with an enhanced diffuse emission. The first possibility is addressed in [8], while the latter will be presented in this contribution.

\section{Neutrinos from the Milky Way}

Neutrinos can be produced close to galactic Cosmic Rays (CR) accelerators such as supernova remnants when high energy protons or nuclei interact with the surrounding matter. A large amount of pions is produced, and the number of produced $\pi^{0}$ is equal to the sum of $\pi^{+}$and $\pi^{-}$. While $\pi^{0}$ immediately decay into a pair of $\gamma$-rays, $\pi^{ \pm}$mesons decay into muons and muon neutrinos. As a consequence, the expected neutrino flux is equal to the $\gamma$ flux of hadronic origin. The energy spectrum of this flux follows the $\mathrm{CR}$ spectrum at the acceleration site $-\mathrm{E}^{-\Gamma}$ with $\Gamma=2.0$ for Fermi acceleration scenarios [9] - since the decay usually takes place before the primary particle can interact.

Another neutrino contribution from the Milky Way is expected from CRs propagating in the inner region of our Galaxy. A CR can interact with the dense environment, producing $\gamma$-rays and neutrinos. Data from the Fermi/LAT detector provide the best observation of this diffuse $\gamma$ flux in the Galactic Plane [10], though no observation of the neutrino counterpart is available.

Assuming that a certain fraction of the observed diffuse $\gamma$ flux in the central region of the Galaxy originates from hadronic mechanisms, the neutrino yield from CR propagation can be calculated. Different predictions are available such as the ones in $[11,12,13]$. Each of these provides a different description of the expected neutrino flux, with an overall normalisation that can vary 
by one order of magnitude. A rather hard spectrum is expected, described, at least in part of the energy range, by a broken power law with spectral index $\Gamma \sim 2.4 \div 2.5$.

\section{The IceCube signal}

The IceCube cosmic neutrino signal was initially [3] fitted by a broken power law spectrum with spectral index $\Gamma$ equal to 2 :

$$
E^{2} \frac{d \Phi}{d E}=(0.95 \pm 0.3) \times 10^{-8} \mathrm{GeV} \mathrm{s}^{-1} \mathrm{sr}^{-1} \mathrm{~cm}^{-2} .
$$

while also an $\mathrm{E}^{-2.3}$ spectrum was reported to be compatible with the observed signal, mainly because of the absence of events above $2 \mathrm{PeV}$ and the lack of an enhanced neutrino detection in the region of the Glashow resonance.

The further extension of the IceCube data set and refinements in the analysis for the observation of lower energy neutrinos resulted in a steepening of the energy spectrum; in the most recent publication [6] the best fit is reported to be:

$$
\frac{d \Phi}{d E}=\left(2.06_{-0.3}^{+0.4}\right) \times 10^{-18}\left(\frac{E}{100 \mathrm{TeV}}\right)^{2.46 \pm 0.12} \mathrm{GeV}^{-1} \mathrm{~s}^{-1} \mathrm{sr}^{-1} \mathrm{~cm}^{-2}
$$

for $25 \mathrm{TeV}<\mathrm{E}_{v}<1.4 \mathrm{PeV}$, rejecting the $E^{-2}$ hypothesis with a significance of $3 \sigma$.

This flux is observed as an all sky flux by IceCube. This can be related to the bias in efficiency of the analysis to shower events, for which IceCube has a rather bad angular resolution. However, an accumulation of shower events is present in the IceCube sky map of [3] even if it is not significant because of the poor directional reconstruction. This accumulation could point towards a diffuse emission region of cosmic neutrinos. In addition, as the Southern sky contains (most) of the Galactic Plane, the soft spectrum derived from IceCube measurement and an enhancement of the neutrino flux in the Southern sky hint towards a possible neutrino emission from the Galactic Plane.

\section{Data analysis}

Given the IceCube effective area, the cosmic flux which can produce a certain number of events from a region of the sky with angular size $\Omega \sim 0.1-0.2 \mathrm{sr}$ can be computed as a function of the signal spectral index [5].

Two possible signal regions have been chosen for this analysis. The first one corresponds to a $10^{\circ}$ circular region around the largest accumulation of IceCube signal events (IceCube hotspot). The centre of this circular area is at $1=18^{\circ}$ and $b=-9^{\circ}$ in galactic coordinates. This position has been computed averaging the position of the HESE events weighted according their energy and angular uncertainty.

The second signal region is chosen to represent the Galactic Plane area. A rectangular region having $|1|<40^{\circ}$ and $|\mathrm{b}|<3^{\circ}$ is selected since it encloses the central part of the Fermi/LAT diffuse galactic plane flux. 
In any case, as the background per unit solid angle can be considered rather similar for regions that have similar exposures and since the sensitivity depends on the intensity of the background flux in terms of number of events per steradians, the results are independent on the choice of the signal region.

Data collected by the ANTARES neutrino telescope from 2007 to 2013 have been considered for this analysis.

\subsection{Atmospheric background}

The main background in the search for cosmic neutrinos is given by downward-going atmospheric muons reaching the detector; these events are simulated using the MUPAGE software [15]. Since only neutrinos can traverse the Earth, neutrino telescopes look at upward going events to reject this background. Wrongly reconstructed atmospheric muons, mimicking upgoing neutrino events can be rejected by a selection on the track quality parameter $\Lambda$ and the estimate for the angular error $\beta$.

An irreducible background comes from atmospheric neutrinos coming from the decay of shortlived particles in extensive air showers. The conventional component, coming from the decay of pions and kaons, is described by the Honda et al. flux [16]: in general this conventional flux can be described with a broken power law energy spectrum, with spectral index $\Gamma$ asymptotically going to 3.7.

A prompt component is expected to come from charmed hadrons, which decay in a much shorter time and give a harder neutrino energy spectrum $(\Gamma \sim 2.7)$. The Enberg et al. [17] model is used in this work to parametrise the prompt component. A measurement of the atmospheric neutrino energy spectrum using ANTARES data has been performed and the results are shown in [18].

Since signal and background spectra can be described by power laws with different indices, a cut on the reconstructed energy [19] can provide a selection for cosmic neutrinos, since at high energy the signal flux is naturally enhanced.

\subsection{Background estimation from data}

A signal from a Southern Sky region is searched for by comparing the number of selected events from the chosen on-zones to that of similar regions with no expected signal (off-zones). This choice avoids simulation related biases in the estimation of the signal intensity after event selection.

Off-zones are defined as fixed regions in equatorial coordinates which have identical size and shape as the on-zone but have no overlap with it. In local coordinates, off-zones have the same sidereal-day periodicity as the on-zone and span the same fraction of the sky, but with some fixed delay in time. This approach has been already used in ANTARES to search for events from the Fermi bubbles [20, 21] and in a previous analysis of the Galactic Plane region [22].

Figure 1 reports the position of the signal and background regions in galactic coordinates; also the position of the Fermi Bubbles is shown. Off-zones are shifted in the sky to avoid any overlap with the Fermi bubbles, so that none of the possible signal events from these areas enters in the background estimation. In the case of the IceCube Hotspot, up to 12 off-zones can be used for background estimation, while for the Galactic Plane selection the maximum is 9 off-zones. 


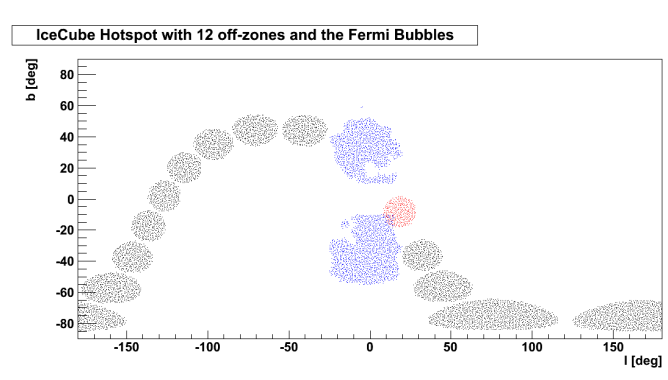

(a) IceCube hotspot

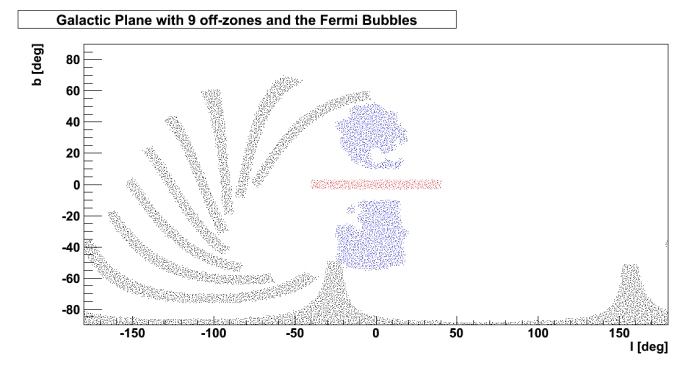

(b) Galactic plane

Figure 1: Signal (red dots) and background (black dots) regions for the on/off-zones of the analysis of: a) the IceCube hotspot; b) the Galactic Plane region. Also shown the shape of the Fermi bubbles (blue dots) as used in the analysis of [21].

While data from the signal regions are blinded until the event selection has not been completely defined, off-zones can be used to estimate the agreement between data and Monte Carlo as well as the relative agreement between data and data from different off-zones. No anomalous behaviour has been found in the analysed data set.

\subsection{Cut optimisation}

The optimisation of the event selection to enhance the possible cosmic signal against the atmospheric background is made on the basis of track quality parameters to reject wrongly reconstructed atmospheric muons and on the energy estimation to select cosmic neutrinos over the atmospheric background. The optimal selection cut, which maximises the sensitivity, is computed on the basis of the Model Rejection Factor (MRF) procedure [23] for the $\Gamma=2.4$ and $\Gamma=2.5$ hypotheses.

The optimal selection cut is:

$$
\Lambda>-5.0, \beta<0.5^{\circ}, E_{A N N}>10 \mathrm{TeV}
$$

where $\Lambda$ and $\beta$ are the track quality parameters described in section 4.1, while $\mathrm{E}_{A N N}$ is the energy estimator from the Artificial Neural Network algorithm presented in [19].

Considering a signal flux with an energy spectrum $\sim \mathrm{E}^{-2.4}\left(\mathrm{E}^{-2.5}\right)$ the $90 \%$ confidence level sensitivity is $2.0(6.0) 10^{-5} \mathrm{GeV}^{-1} \mathrm{~cm}^{-2} \mathrm{~s}^{-1} \mathrm{sr}^{-1}$. For comparison, assuming an $\mathrm{E}^{-2.4}$ spectrum, the normalisation of an IceCube-like flux producing 2 or more events from a region in the sky of 0.1 sr is larger than $2.010^{-5} \mathrm{GeV}^{-1} \mathrm{~cm}^{-2} \mathrm{~s}^{-1} \mathrm{sr}^{-1}$ and any of these scenarios can be rejected. Figure 2 compares the obtained sensitivity in the Galactic Plane region to the expected neutrino flux from CR propagation of [11] and [13]. This sensitivity holds in the energy range $3 \mathrm{TeV}-300 \mathrm{TeV}$, which contains the central $90 \%$ of the expected signal.

\section{Results}

As far as the IceCube HESE hotspot is concerned, the average number of events coming from the chosen off-zones passing the signal selection criteria is 1.0 over the entire period. One event is also observed from the signal region and the measurement is perfectly compatible with the background only expectations. For the Galactic Plane 2.5 events are observed on average for the background regions and one is detected from the on-zone after the final selection. An underfluctuation 


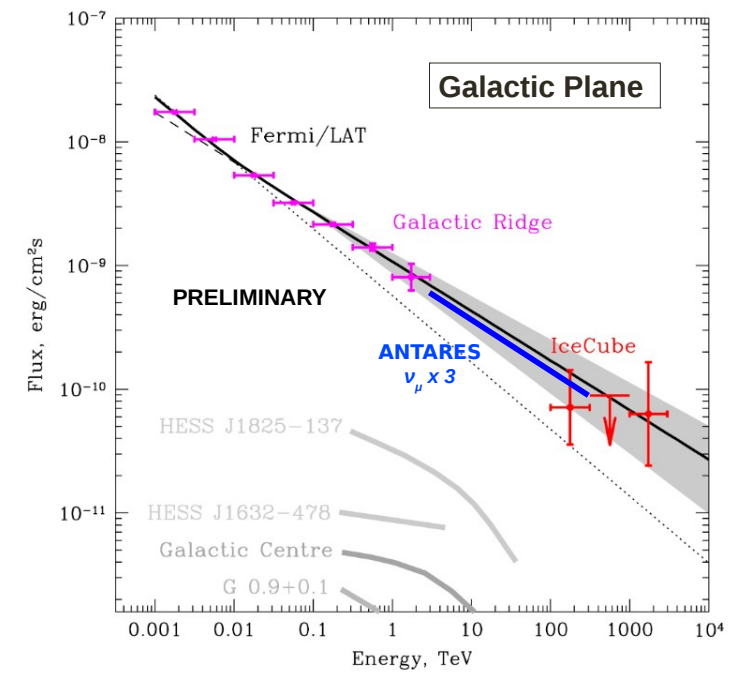

(a)

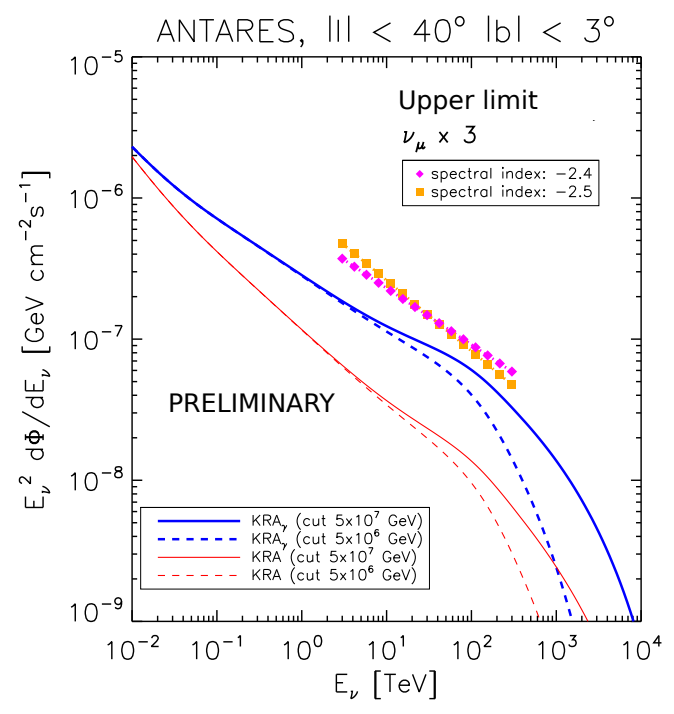

(b)

Figure 2: a) ANTARES sensitivity for the Galactic plane region assuming neutrino spectral index $\Gamma=2.4$ (blue line) compared to theoretical expectations and experimental data from Fermi/LAT and IceCube as computed in [11]; b) $90 \%$ C.L. upper limit for signal spectrum $\mathrm{E}^{-2.4}$ (magenta dots) and $\mathrm{E}^{-2.5}$ (orange dots) for the null observation of this analysis compared to the expected neutrino flux from the simulations presented in $[13,14]$.

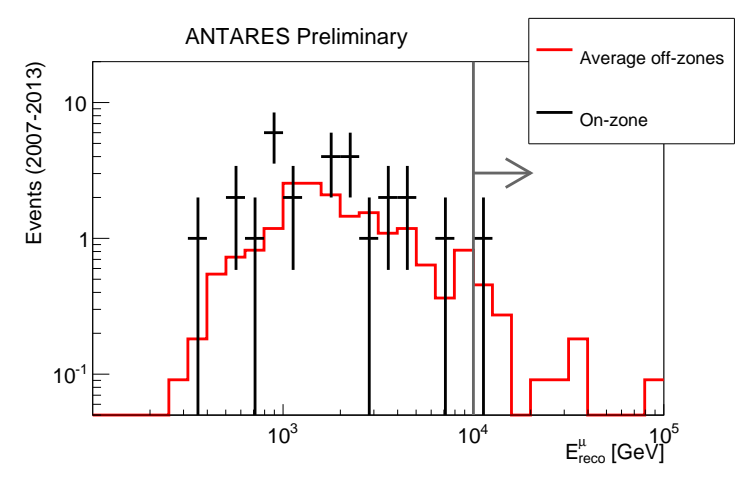

(a) IceCube HESE hotspot

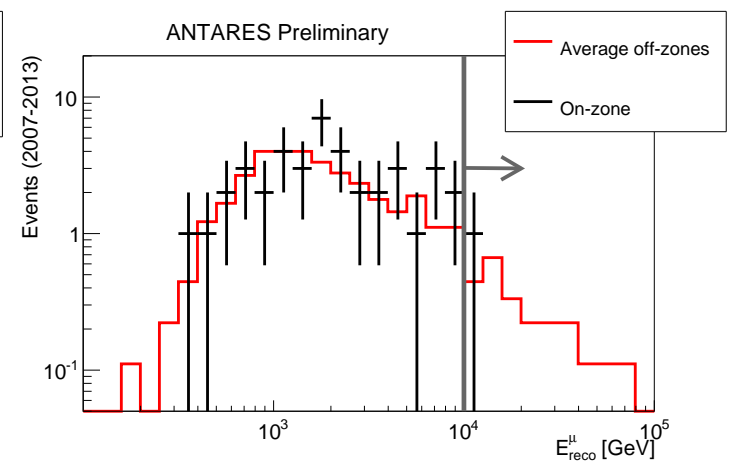

(b) Galactic Plane

Figure 3: Reconstructed energy distribution of events in the signal (black crosses) and background (red line) regions for a) the IceCube hotspot region, b) Galactic Plane. The gray line shows the energy selection cut applied in the procedure. No significant excess is observed at high energy.

of the background is thus present in the signal region. The reconstructed energy distributions of events for the on and off-zones are reported in figure 3.

Neither the hotspot region nor the Galactic Plane area present an excess of events with respect to the background only evaluation. For this reason an upper limit at $90 \%$ confidence level on the signal flux can be set and it corresponds to the ANTARES sensitivity for the analysed spectral indexes. The obtained upper limits are reported in figure 4 compared to the expected neutrino flux that would induce a certain number of IceCube HESE events. These fluxes have been computed 


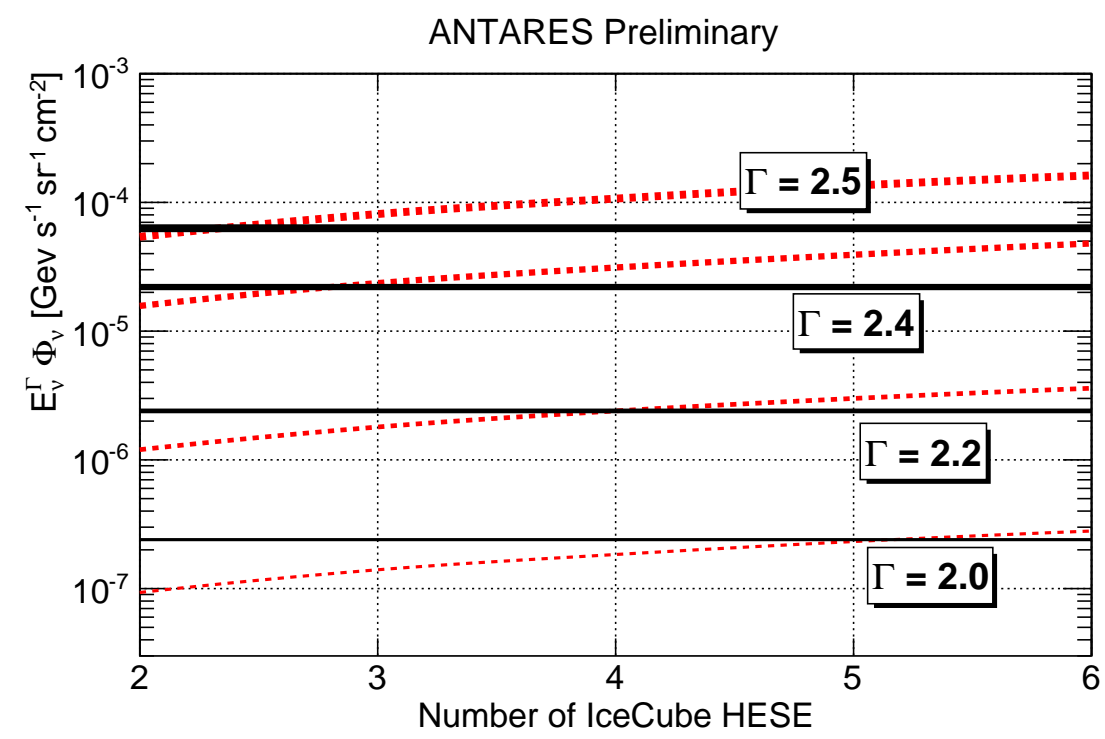

Figure 4: Upper limits coming from the null observation in the Galactic Plane region compared to the expected flux producing a certain number of IceCube HESE events. Selection cuts are optimised for $\Gamma=2.4$ and 2.5, while the limits for harder spectral index are computed with non optimal selection.

on the basis of the effective areas reported in [3]. Any model producing more than 3 events in the IceCube HESE sample from the Galactic Plane region is excluded at $90 \%$ confidence level for a spectral index larger than 2.4. The upper limits for harder spectral assumptions with the same selection criteria are also shown.

The lack of signal events in ANTARES data significantly constrains the possible Galactic origin of the IceCube Southern sky excess. Adding cascade events in the analysis is foreseen. For these events the angular resolution of ANTARES is much better than that of IceCube [24] and the combined search in track and showers can improve the possiblity to observe such a diffuse flux from our Galaxy and to explain the Southern sky excess in IceCube data.

\section{References}

[1] C. James (for the ANTARES Collaboration) Proceedings of the $34^{\text {th }}$ ICRC, The Hague, Netherlands (2015).

[2] M.G. Artsen et al., Science 342: 1242856 (2013).

[3] M.G. Artsen et al., Phys. Rev. Lett. 113: 101101 (2014).

[4] M.G. Artsen et al., Phys. Rev. Lett. 114: 171102 (2015)

[5] M. Spurio, Phys. Rev. D 90: 103004 (2014).

[6] M.G. Artsen et al., Phys. Rev. D 91: 022001 (2015).

[7] T.K. Gaisser et al., Phys. Rev. D 90: 023009 (2014).

[8] J. Barrios Martí (for the ANTARES Collaboration), Proceedings of the $34^{\text {th }}$ ICRC, The Hague, Netherlands, ID 1077 (2015).

[9] E. Fermi, Phys. Rev. 75: 1169 (1949); E. Fermi, Astroph. J. 119: 1 (1954). 
[10] M. Ackermann et al., ApJ 750: 3, (2012).

[11] A. Neronov, D. Semikoz, C. Tchernin, Phys. Rev. D 89: 103002 (2014); A. Neronov, D. Semikoz, arXiv:1412.1690 (2014).

[12] Y.Q. Guo, H.B. Hu \& Z. Tian, arXiv:1412.8590 (2014).

[13] D. Gaggero et al., arXiv:1504.00227 (2015).

[14] A. Marinelli et al., Proceedings of the $34^{\text {th }}$ ICRC, The Hague, Netherlands, ID 1126 (2015).

[15] G. Carminati et al., Computer Physics Communications 179, 12: 915-923 (2008).

[16] M. Honda et al., Phys. Rev. D 75: 043006 (2007).

[17] R. Enberg et al., Phys. Rev. D 78: 043005 (2008).

[18] S. Adrián Martínez et al., Eur. Phys. J. C 73: 2606 (2013).

[19] J. Schnabel, Nucl. Instr. and Meth. A 725:106-109 (2013).

[20] S. Adrián Martínez et al., Eur. Phys. J. C 74: 2701 (2014).

[21] S. Hallmann (for the ANTARES Collaboration), Proceedings of the $34^{\text {th }}$ ICRC, The Hague, Netherlands, ID 1059 (2015).

[22] E. Visser (for the ANTARES Collaboration), Proceedings of the 24th ECRS, Kiel, Germany (2014).

[23] G.C. Hill \& K. Rawlins, Astrop. Phys. 19: 393 (2003).

[24] T. Michael (for the ANTARES Collaboration), Proceedings of the $34^{\text {th }}$ ICRC, The Hague, Netherlands, ID 1078 (2015). 